\title{
Il valore sociale dei rifiuti. L'intreccio tra istituzioni e pratiche di recupero nello spazio urbano di Casablanca (Marocco)
}

Waste social value. Links between institutions and waste recovery practices in the urban space of Casablanca (Morocco)

\section{Anna Karin Giannotta}

\section{OpenEdition}

\section{Journals}

Edizione digitale

URL: http://journals.openedition.org/aam/3377

DOI: $10.4000 /$ aam.3377

ISSN: 2038-3215

\section{Editore}

Dipartimento Culture e Società - Università di Palermo

Notizia bibliografica digitale

Anna Karin Giannotta, «Il valore sociale dei rifiuti. L'intreccio tra istituzioni e pratiche di recupero nello spazio urbano di Casablanca (Marocco)», Archivio antropologico mediterraneo [Online], Anno XXIII, n. 22 (2) | 2020, online dal 20 juin 2020, consultato il 29 janvier 2021. URL: http://journals.openedition.org/ aam/3377 ; DOI: https://doi.org/10.4000/aam.3377

Questo documento è stato generato automaticamente il 29 janvier 2021.

\section{c) (†)}

Archivio antropologico mediterraneo è distribuita con Licenza Creative Commons Attribuzione - Non commerciale - Non opere derivate 4.0 Internazionale. 


\title{
Il valore sociale dei rifiuti.
} L'intreccio tra istituzioni e pratiche di recupero nello spazio urbano di Casablanca (Marocco)

\author{
Waste social value. Links between institutions and waste recovery practices in \\ the urban space of Casablanca (Morocco)
}

Anna Karin Giannotta

\section{Introduzione}

1 Casablanca, città tentacolare e polmone economico del Marocco. Arrivando dalla stazione ferroviaria di Casa-Port, prendendo un grand-taxi e attraversando le strade trafficate della città, la vista di cassonetti dell'immondizia strabordanti e di oggettirifiuti abbandonati sui cigli delle strade risulta una costante. Frequentemente si può incrociare il proprio cammino con quello dei recuperatori di rifiuti, i quali trainando il loro carretto, la karoussa, si muovono agilmente attraversando la città, selezionando e raccogliendo materiali potenzialmente adatti alla vendita e al riciclo. Alla luce dell'interpretazione dei dati raccolti durante la ricerca etnografica, è emerso un intreccio tra pratiche e politiche ufficiali e ufficiose di recupero degli scarti e una flessibilità nell'attribuzione di un determinato valore all'oggetto rifiuto.

2 Sin dai primi resoconti storiografici lo scarto - che si materializzava principalmente come deiezione umana e animale o come materiale in eccesso prodotto dalle attività di cucine, botteghe e mercati - è stato oggetto di rappresentazioni basate su un 'principio di allontanamento' (Pinna 2011; Viale 1994). La rivoluzione industriale del XVIII secolo segna un punto di svolta: la ricerca economico-sociale riserva la propria attenzione da un lato all'analisi dei processi di urbanizzazione e delle alterazioni dei modi di produzione e consumo che hanno trasformato villaggi e piccole città in metropoli 
(Marx 1867, Simmel 1903), dall'altro all'emergere di nuovi mestieri come quello dello chiffonnier ${ }^{1}$ (Compagnon 2017). A partire dagli anni '90 la letteratura prodotta sul recuperatore di rifiuti si moltiplica, delineando i tratti di un attore sociale che, qualora presente, si rivela centrale nelle filiere delle grandi città contemporanee. A seconda delle varie località assume denominazioni specifiche: i cartoneros argentini (Vergara 2008), i pariah indiani (Baud, Schenk 1994), gli zabbâleen egiziani (Debout, Florin 2010) o i binners nordamericani (Raoulx et al. 2009). Parallelamente si sviluppa un discorso impostato sulle politiche pubbliche volte all'integrazione dei recuperatori nelle economie circolari e formali della gestione dei rifiuti. I recenti studi hanno affrontato tale tematica da una prospettiva ecologista, economica e umanitaria, rilevando e descrivendo le dinamiche prodotte dal contatto tra sfera informale e formale (Medina 2007; Schamber, Suarez 2007). Recenti studi sull'informalità nel Nord e nel Sud del Mondo hanno dedicato particolare attenzione alla precisazione della nozione ${ }^{2}$, spesso e frettolosamente definita come l'insieme di tutte quelle attività caratterizzate dall'assenza di una regolamentazione governativa o statale (Lautier 1991). Nel presente articolo verrà utilizzata come categoria complementare alla formalità per mettere in luce i diversi collegamenti che caratterizzano il contesto urbano di Casablanca e sottolineare il ruolo dello Stato (e di un'attitudine oscillante tra repressione, controllo e laisser faire) e della pressione burocratica nella produzione di rappresentazioni e meccanismi legati a un distanziamento rispetto all'inquadramento istituzionale (Saitta et al. 2013; Guha-Khasnobis et al. 2006). Lo studio di Tim Cresswell (1996) sulla nozione di trasgressione in relazione allo spazio è un altro contributo al tema dell'informalità. Secondo il punto di vista dell'autore esiste una stretta correlazione tra comportamento trasgressivo e devianza quando entrambi avvengono in violazione di norme e regole stabilite da gruppi dominanti; si adoperano in tal caso argomentazioni su sporcizia, immondizia, inquinamento e oscenità per designare attività che sono out-of-place, fuori luogo rispetto a un dato scenario normativo.

3 Infine, negli ultimi trent'anni una prospettiva locale e transnazionale ha avuto un ruolo decisivo nel definire il rifiuto come oggetto di politiche volte a limitarne l'impatto ambientale tramite riciclo e smaltimento (Gilbert, Henry 2012; Cavé 2015). Sono emersi così nuovi attori coinvolti in questo processo di valorizzazione appartenenti al settore privato: società multinazionali specializzate nello smaltimento dei materiali e dei rifiuti). A partire da queste considerazioni il presente studio intende esplorare i significati attorno all'oggetto-rifiuto, alle pratiche e agli attori ad esso associati nella sua dimensione relazionale, con un focus sul fitto tessuto economico e sociale della città. In quest'ottica mi ispirerò alla teoria elaborata da Arjun Appadurai secondo il quale l'oggetto abbandonato, passando nelle mani di diversi individui, cambia il suo 'regime di valore' (1986): viene così reperito, ripescato, spostato da un luogo all'altro e reintegrato in un ciclo di scambio. L'articolo nel complesso porrà in rilievo l'intreccio tra sfera istituzionale e sfera non istituzionale nella (in)visibilità ${ }^{3}$ (Mubi Brighenti 2010) dei processi di gestione, recupero e riciclo dei materiali di scarto a Casablanca. Il termine istituzionale viene utilizzato nel presente saggio in riferimento a strutture di governo specifiche in grado di produrre un discorso dominante in un determinato ambito. Le istituzioni e i loro attori contribuiscono in tal senso a formalizzare la loro voce e il loro orientamento politico su questioni specifiche (Douglas 1986; Herzfeld 2006), come nel suddetto caso la gestione dei rifiuti. Secondo i dati emersi durante il mio fieldwork rientrano nella categoria di istituzione la Monarchia, il Parlamento e i suoi agenti (Ministero dell'Ambiente, forze militari e di sicurezza ecc...), organizzazioni 
internazionali come la Banca Mondiale e tutte quelle entità che servendosi dell'apparato normativo e legislativo in vigore dettano le norme sulle pratiche di recupero e di riciclo. Centrale risulterà il mestiere degli chiffonniers marocchini, che sarà analizzato portando al dialogo la nozione di 'valore sociale' (Appadurai 1986) del rifiuto, di 'giustizia socio spaziale' (Soja 2010; Lefebvre 1974) e di 'informalità' ed economia informale ${ }^{5}$ (Hart 1973; Chen 2012).

\section{Metodologia e campo}

4 Ho vissuto a Casablanca per circa nove mesi, da settembre 2016 a maggio 2017, svolgendo uno stage presso il Consolato generale d'Italia e l'ICE (l'Istituto Nazionale per il Commercio Estero) e dedicandomi parallelamente alla ricerca sul campo. Grazie alle sollecitazioni che inizialmente mi sono venute rispettivamente dall'allora senior trade analyst e dal Direttore Generale dell'ICE, ho iniziato a interessarmi alla gestione dei rifiuti in Marocco in un'ottica istituzionale. L'apparato legislativo in materia di rifiuti risulta imponente e al pari dell'arsenale di organizzazioni locali e internazionali operanti in modo congiunto e/o parallelo in tale scenario ${ }^{6}$. Pertanto una prima fase di revisione delle fonti è stata fondamentale per una conoscenza preliminare della normativa e degli attori che dominano lo scenario politico e istituzionale preso in esame. Tra i principali documenti ufficiali consultati spiccano la Loi 28.00, la legge che regola le azioni da perseguire in materia di gestione dei rifiuti e il Programme National des Déchets Ménagers assimilés (PNDM) ${ }^{7}$ promosso a partire dal 2007 dal Dipartimento per l'Ambiente e il Ministero dell'interno con l'appoggio della Banca Mondiale. La 'Legge 77-15' impone il divieto di produzione, importazione, esportazione, commercializzazione e utilizzo di sacchi in plastica non biodegradabile e connessa a quest'ultima vi è la campagna di sensibilizzazione Zero mika (letteratamente 'zero plastica') lanciata a partire dal 2016 dalla Coalition marocaine pour la justice climatique a seguito di una imponente mediatizzazione operata dai canali televisivi nazionali (MEDI 1 TV33, 2M TV) e dall'Agenzia di stampa marocchina (MAP) con il supporto della stampa digitale e dei social networks.

5 La ricerca sul campo ha seguito così diverse fasi, alcune di loro concomitanti: la lunga permanenza a Casablanca ha favorito un'osservazione partecipante quotidiana delle pratiche di selezione e di raccolta dei rifiuti da parte dei recuperatori informali. In modo particolare, l'osservazione è stata svolta in quei quartieri che per motivi di lavoro frequentavo quotidianamente: Bourgogne e Gauthier. Ho svolto parte della ricerca collaborando e accompagnando un responsabile di zona della ditta Ozone Environnement di Salé (una delle imprese private che detiene circa sessanta contratti di gestione di nettezza urbana sul territorio nazionale marocchino), Hamid ${ }^{8}$, nei suoi giri di perlustrazione quotidiani, dove era «necessario un intervento di Ozone per tenere sotto controllo l'attività dei recuperatori informali, i quali lasciano sempre i rifiuti sparsi attorno i cassonetti dell'immondizia»'. Grazie all'incontro con un professore e ricercatore di Geografia culturale presso l'Università Hassan I di Khouribga, ho preso parte in quanto discussant a un seminario su La gouvernance des déchets urbains: enjeux territoriaux et sociaux, tenutosi il 12 e 13 aprile 2017 a Rabat. In quell'occasione ebbi la possibilità di conoscere Sidi, grossista e proprietario di una goulssa, uno dei tanti centri di stoccaggio di rifiuti ubicati nelle zone periferiche di Casablanca. Da quel momento il campo si è spostato anche all'interno della goulssa, situata a Lahraouine, un quartiere 
periferico di Casablanca. La modalità di raccolta dei dati si è differenziata per ognuno di questi microcontesti. Svolgere un'etnografia in strada ha implicato specifiche modalità di osservazione caratterizzate da un movimento continuo. Le annotazioni sul diario di campo venivano trascritte mnemonicamente durante i miei spostamenti. Le conversazioni si differenziano principalmente in base al tipo di interlocutori e al registro linguistico utilizzato (lingua francese o dialetto marocchino dārija). In quest'ottica la conoscenza del dārija ha favorito l'interazione con quella fascia di interlocutori che, come nel caso dei recuperatori informali, raramente ha studiato il francese e quindi lo utilizza correntemente.

\section{Quadro normativo e pratiche informali a Casablanca}

Nel giro di circa trent'anni, Casablanca è passata dallo status di villaggio di pescatori a quello di metropoli, con oltre 4 milioni di abitanti ${ }^{10}$ (HCP, 2014). Questo mutamento ha interessato anche un aspetto visibile e materiale della città: la produzione di beni e merci, il loro consumo, e il conseguente accumulo di rifiuti. Il loro valore potenziale ha attirato l'attenzione di investitori e finanziatori internazionali i quali, lungimiranti, hanno visto del profitto nel sistema di gestione dei rifiuti della città. La preoccupazione e l'interesse pubblico e politico del Marocco verso la questione dei rifiuti, si sono manifestati pubblicamente solo negli anni 2006-2007 con due importanti azioni politiche e legislative: l'adozione della legge $28-00^{11}$ e di un quadro normativo concernente la gestione e lo smaltimento dei rifiuti e la promozione di un Programme National des Déchets Ménagers (PNDM). Per adattarsi alle spinte provenienti dalle istituzioni internazionali e al rapido sviluppo demografico e socio economico con conseguente deterioramento dell'ambiente, il Ministero dell'ambiente marocchino ha recentemente adottato la legge 28.00 relativa alla gestione dei rifiuti e al loro smaltimento (Bulletin Officiel 2006). Tra gli obiettivi dichiarati nel testo di legge ritroviamo la prevenzione e la riduzione della produzione di rifiuti, la creazione di una filiera di selezione-riciclaggio-valorizzazione al fine di ottenere materiali riutilizzabili o energia e la realizzazione di un sistema di controllo delle infrazioni commesse in questo ambito (Ibidem). Su quest'ultimo punto spicca il testo dell'art. 70 che afferma:

Chiunque - al di fuori dei luoghi a tal fine designati - deposita, getta o interra rifiuti ritenuti pericolosi [...] o ne effettua lo stoccaggio, il trattamento, l'eliminazione o l'incenerimento è soggetto a una multa da 10.000 a 2.000 .000 di dirham ${ }^{12}$ e alla reclusione da 6 mesi a 2 anni (Ibidem).

Inoltre nell'art.3 il rifiuto viene definito come:

[...] qualsiasi residuo derivante da un processo di estrazione, sfruttamento, trasformazione, produzione, consumo, utilizzo, controllo o filtraggio, ed in generale, qualsiasi oggetto e materiale abbandonato o che un soggetto elimina al fine di non arrecare danno alla salute ed alla sanità pubblica e dell'ambiente (Ibidem).

8 L'intento dichiarato nel testo normativo è dunque la creazione di una filiera di valorizzazione e riciclo, attraverso una minuziosa distinzione degli stessi sulla base della loro natura: rifiuti domestici, industriali, medici, ospedalieri e farmaceutici, pericolosi, agricoli, finali e biodegradabili. Adottato nel 2007, il PNDM aspira a un rinnovamento del sistema tramite la cosiddetta Gestion Déléguée $e^{13}$ (Banca Mondiale, 2010). Avvalendosi della 'Legge 54-05' del 2016, relativa alla gestione delegata dei servizi pubblici, si concretizza la realizzazione dei Partenariati Pubblico-Privati. I 
cosiddetti PPP assegnano a multinazionali straniere private la gestione della nettezza urbana e del trattamento dei rifiuti di quartieri o intere città. Vediamo nello specifico come si incastrano queste misure nel tessuto sociale del Marocco e nello specifico di Casablanca.

9 In base ai punti principali del quadro normativo presentato, ad oggi il $70 \%$ dei comuni in Marocco ha scelto di privatizzare la gestione dei rifiuti solidi urbani (Ghalloudi et al, 2015: 242). Nello specifico a Casablanca, sino al 2017 l'«80\% della nettezza urbana era affidata a due enti privati: Sita Blanca (filiale di Suez environnement) e Averda (multinazionale libanese), mentre il 20\% della superficie della città non godeva di alcun servizio di raccolta dei rifiuti» (Florin 2015:4). Tuttavia, sia la stampa che i cittadini e le stesse autorità comunali hanno ben presto riconosciuto i difetti di un'amministrazione decentrata di un tale servizio pubblico. In Marocco, l'attuazione delle varie politiche e disposizioni legali è responsabilità di diverse e numerose istituzioni pubbliche, carenti di cooperazione e coordinamento delle attività e reciproca informazione (Zaouaq, Zaouaq 2019). A questo si aggiunge un livello piuttosto scarso e limitato della capacità di recupero e riciclo dei materiali, nonché nell'inadempienza degli obiettivi prefissati in materia di smaltimento dei rifiuti. Nonostante i buoni propositi e gli strumenti all'avanguardia messi in campo (come i chip elettronici posizionati su ogni camion della nettezza urbana), la città continua a combattere contro una mala gestione e riciclo dei materiali di scarto e il malcontento della popolazione cresce di giorno in giorno. Casablanca viene spesso definita come casa-negra o la ville poubelle (la città spazzatura) ${ }^{14}$ dai suoi abitanti e dalla stampa, in riferimento alla presenza costante di cassonetti strabordanti di rifiuti e di un odore nauseabondo proveniente da diversi punti della città dove sorgono discariche selvagge (Paillard 2019). Nel mirino delle accuse l'operato di Sita Blanca e le molteplici inadempienze del capitolato d'oneri, come ad esempio ritardi e mancanze nella raccolta, nel trasporto dei rifiuti verso la discarica e nella pulizia delle strade pubbliche della città (Ghalloudi et al., 2015: 255-256). Questa situazione di 'degrado della proprietà', ha spinto il sindaco della città a indire nel settembre 2017 un consiglio speciale finalizzato alla recessione (avvenuta il 16 settembre 2017) del contratto di appalto con la società Sita Blanca ${ }^{15}$. L'organizzazione territoriale decentralizzata crea una distribuzione dei ruoli e dei poteri spesso non chiara (Zaouaq, Zaouaq 2019). Infatti, accanto all'istituzione del governo e del re sovrano Mohammed VI, capo religioso, politico e militare, esiste quella del Makhzen:

uno spazio politico dai contorni sfumati, una specie di governo ombra che al proprio interno esprime e compone tutti i conflitti, sotto l'occhio vigile del re e al tempo stesso tutela la supremazia della monarchia attraverso meccanismi di arbitraggio, clientelismo e condizionamento (Emiliani 2002: 172).

Come vedremo nella sezione 4 alcune figure del Makhzen sono emerse anche durante la ricerca sul campo, in riferimento al controllo operato dal cosiddetto moqaddem sul territorio su cui si estende la goulssa di Sidi ${ }^{16}$.

\section{Gli attori e i luoghi: i bouâara e le goulssa tra pratiche di riconoscimento e di riappropriazione dello spazio urbano}

11 Il primo anello della filiera di gestione e smaltimento di rifiuti in Marocco è rappresentato dai recuperatori informali, anche chiamati chiffonniers in francese o 
bouâara e mikhala in dialetto marocchino ${ }^{17}$. Dopo una prima fase di recupero dei rifiuti, il materiale selezionato viene poi venduto ai grossisti e semi grossisti ${ }^{18}$, dei veri e propri business-men dei rifiuti i quali disponendo di una determinata somma di capitale, sono (o sono stati) in grado di acquistare macchinari (trituratore di plastica, compattatori ecc.), mezzi di trasporto (camion o furgoni) e magazzini o spazi adibiti allo stoccaggio di rifiuti, le cosiddette goulssa ${ }^{19}$ o i petit dépôt (piccoli garage). Per facilitare la comprensione etnografica degli spazi citati, si tenga presente che la ricerca ha avuto inizio a Bourgogne e Gauthier, quartieri dove ho iniziato a seguire la circolazione dei rifiuti nel momento in cui vengono abbandonati per poi essere selezionati e recuperati dagli chiffonniers marocchini. La fase intermedia dei pétit dèpots ha avuto luogo nei pressi della medina della città, a circa un chilometro e mezzo a nord dai due quartieri. La goulssa invece è situata nel quartiere periferico di Lahraouine distante circa dodici chilometri a sud-est rispetto alle zone centrali della città (per l'appunto Bourgogne e Gauthier). Infine l'ultima tappa è quella di Sidi Maârouf, quartiere situato tra l'aeroporto internazionale Mohamed V ed il centro di Casablanca.

L'attività dei bouâara comprende la ricerca, la selezione, la raccolta, il trasporto e la rivendita dei rifiuti. Si muovono per le strade trafficate della città con agilità, trasportando sulle spalle un carretto, la karoussa (o anche chiamata charrette in francese) che può arrivare a pesare fino a 500 chilogrammi di materiale. «La charrette ed il suo utilizzo sono stati proibiti nel 1975. [...] Oggi questi veicoli sono semplicemente 'tollerati' e non sono soggetti ad alcun controllo, di qualsiasi natura esso sia. Se ne ignora persino il numero» (Godard, Fatonzoun 2002:346). Questo dato è stato confermato durante la ricerca sul campo. Riporto di seguito uno stralcio di intervista a Zakarya, un interlocutore conosciuto durante il fieldwork:

Oggi giorno chiunque può dotarsi di un carretto. Basta avere un animale e saperselo costruire. Le karoussa non necessitano di alcuna autorizzazione, non sono assicurate e in caso di incidente i passeggeri non sono in alcun modo indennizzati ${ }^{20}$.

L'esempio dell'ordinanza che vieta la circolazione e l'utilizzo delle karoussa, la conseguente e quotidiana trasgressione della stessa rivelano un atteggiamento di controllo, laisser faire e repressione allo stesso tempo ${ }^{21}$.

Vado ovunque con la karoussa perché la Police ci lascia agire indisturbati, finché ci comportiamo bene. Se lasciamo rifiuti per strada o sporchiamo le strade e i marciapiedi [...] allora può capitare che ci venga sequestrata o ci venga fatta una multa $^{22}$.

14 La capacità di adattamento e la flessibilità delle pratiche degli chiffonniers si manifesta in altre tattiche che vengono messe in atto e riprodotte nello spazio urbano di Casablanca, per far fronte al divieto di utilizzo della charrette. Per evitare che le forze di sicurezza la confischino e per evitare insulti e sguardi intimidatori, i recuperatori marocchini preferiscono muoversi per le strade della città durante le ore notturne. «Riescono a percorrere dai dieci ai trenta chilometri a piedi, lavorando fino a dodici ore ininterrottamente» (Florin 2015: 12). Si dimostrano scaltri nel passare in rassegna determinate zone della città prima del passaggio del camion della nettezza urbana, approfittando dunque della disponibilità di rifiuti prima che questi vengano raccolti. La raccolta avviene in modo discreto, rapido e silenzioso, nel trambusto della città e raramente si verificano interazioni con il resto degli abitanti. Quando questo avviene, vengono rimproverati per aver sparpagliato i rifiuti attorno ai bidoni della spazzatura, vengono rimproverati per la 'sporcizia' ed il disordine che provocano. Svolgono la loro attività singolarmente e in maniera autonoma decidono quali sono i percorsi e le rotte 
da seguire durante la loro giornata lavorativa. Il loro guadagno economico è giornaliero, e dipende dal tipo e dalla quantità di materiali che riescono a raccattare nell'arco di una giornata e a rivendere: «Una volta ho trovato del rame giallo in un edificio abbandonato vicino Boulevard D'Anfa. Quello è il materiale che più ti viene pagato. Era mattina e il pomeriggio non ho lavorato perché la giornata era andata bene ${ }^{23}$ ». Il rame è tra i materiali più redditizi che viene pagato circa $€ 2,50 / \mathrm{kg}$. A titolo comparativo la plastica viene venduta a circa $€ 0.20 / \mathrm{kg}$.

Gli chiffonniers raramente intrattengono rapporti diretti con i grossisti, sia perché raggiungere una goulssa significherebbe percorrere diversi chilometri ed esporsi a possibili incontri spiacevoli che metterebbero a rischio il frutto di una dura giornata di lavoro (ricordiamo il divieto di utilizzo della karoussa), sia perché esistono dei luoghi intermedi. Sono i cosiddetti dépôt o petit dépôt. Il termine, preso dal francese dépôt ed utilizzato anche in dārija, significa letteralmente deposito, magazzino. Costituiscono dei luoghi intermedi, dei garage privati, disseminati nel centro della città, dove gli chiffonniers marocchini depositano il materiale raccolto e lo vendono ai cosiddetti moul al-dépôt, i proprietari del magazzino. Una volta venduto ai moul-al-dépôt, il materiale verrà ulteriormente selezionato per essere poi stipato in appositi mezzi di trasporto, capaci di contenerne una certa quantità, destinati allo scarico nelle goulssa. Si compie così un'ulteriore cernita separando gli scarti in base al tipo di materiali che li compongono. I proprietari di questi centri intermedi di vendita e stoccaggio dispongono di una disponibilità economica tale da permettersi un mezzo di trasporto adeguato al ruolo svolto all'interno di questa filiera. Questo dato ci offre ulteriori informazioni sulle specializzazioni professionali: ai piani alti della scala gerarchica sopra menzionata vi è chi, possedendo una certa somma di capitale, ha a disposizione macchinari (quali trituratore di plastica, compattatori ecc.) o mezzi di trasporto (dalla karoussa ai camion o furgoni). Questi ultimi sono gli strumenti che conferiscono 'visibilità' alle attività di recupero e riciclo dei rifiuti in Marocco. La loro vivace circolazione ci informa sull'estensione di questo tipo di attività nella città. Conferisce agli attori (semi) informali uno spazio di legittimità entro il quale potersi muovere, agire e entrare in un reciproco processo di riconoscimento con i titolari della governance pubblica, ma allo stesso tempo evidenzia diseguaglianze socio professionali tra i diversi attori coinvolti nella filiera analizzata.

Come abbiamo già anticipato precedentemente, in una fase preliminare il rifiuto viene recuperato per poi essere rivenduto a grossisti e semi-grossisti. Viene trasportato così nella goulssa, veri e propri centri di selezione e differenziazione solitamente situati in periferia (Florin 2015). La goulssa di Sidi si trova nel quartiere di Lahraouine. È conosciuto per essere la bidonville dei mikhala in riferimento alla sua posizione in prossimità del quartiere di Sidi Moumen che ospitava sino a qualche anno fa una discarica a cielo aperto, non a norma. Il centro di stoccaggio dei rifiuti raccolti dai recuperatori informali risulta dissociato dal resto dello spazio urbano visibile ed accessibile. Difatti oltre una evidente lontananza geografica dal centro, un recinto di alte barriere in lamiera arrugginita, teli di plastica, assi e tavole di rifiuti secchi compattati accentua questa forma di segregazione spaziale e di allontanamento della povertà dal centro della città. L'isolamento non è solo imposto dai poteri alti come vedremo, ma è simbolo della volontà stessa di recuperatori informali, grossisti e semi grossisti di rimanere al riparo da occhi indiscreti e non mescolarsi con la sfera formale della filiera. Qui, «l'ecosistema più immediato è costituito dai rifiuti degli altri» 
(Piasere 1991: 159): l'oggetto-rifiuto non si configura solo come un bene con un determinato valore economico da predisporre alla vendita delle imprese private, ma costituisce il materiale stesso di costruzione di una determinata forma di abitare. «Tutto qui è costruito con scarti di diversi materiali ${ }^{24} »$. Vi è un intenso brulichio di materiali e persone, ognuno con il proprio ruolo e la propria posizione definita da regole interne. Le karoussa e i moto-furgoncini che raggiungono questo luogo quotidianamente, vi scaricano il materiale: il proprietario (il grossista), in questo caso Sidi, con l'aiuto dei bouâara che lavorano per conto suo, ne valuta il valore ed il peso. Avviene così una prima forma di transazione commerciale.

17 In un spazio adiacente avviene invece un altro processo che trasforma l'oggetto-rifiuto in un materiale dalle caratteristiche adeguate alla vendita presso le grandi imprese private che si occupano di riciclaggio: questo processo comprende la pulizia, la triturazione e la pressatura. "Questo processo serve a economizzare lo spazio e a facilitare lo stoccaggio ed il compattamento dello stesso per una migliore resa ed una migliore vendita ${ }^{25}$ ». Una volta ridotto il volume, il materiale triturato viene raccolto in enormi bustoni di plastica. Questi verranno poi caricati su dei camion, di proprietà dei grossisti della goulssa, e trasportati fino alle imprese private che si occupano del riciclo dei rifiuti in Marocco. L'ultima tappa del rifiuto prevede quindi la vendita alla sfera formale di questa filiera che brulica in ogni momento di persone e oggetti. Sebbene non mi sia mai stato fatto il nome delle industrie private cui Sidi vendeva i materiali lavorati, consultando internet è possibile risalire a molte di loro e alla zona in cui sono localizzate. La maggior parte si trova nel quartiere Sidi Maârouf di Casablanca, centro commerciale e finanziario della metropoli. La filiera riemerge così allo scoperto e dalla periferia ritorna al centro della città ${ }^{26}$.

Le goulssa generalmente non dispongono dei servizi basilari come acqua e elettricità ${ }^{27}$. Per soddisfare le necessità di lavorazione dei materiali, Sidi ha acquistato un gruppo elettrogeno alimentato a benzina, favorendo così l'utilizzo dei trituratori. La precarietà dello status di occupazione delle goulssa non impedisce che queste vengano comunque riconosciute dai poteri pubblici (Florin 2015). A riprova di questo 'atto di riconoscenza' vi è uno dei ruoli assegnati al moqaddem, il quale spesso e volentieri abusa del suo potere, imponendo ai proprietari delle goulssa e ai bouâara determinate regole di comportamento in prossimità dei cassonetti della spazzatura e talvolta anche richiedendo forme di tangenti per compensare la loro occupazione illegale del territorio: «Siamo in guerra con loro perché sia noi che loro siamo proprietari di questo pezzo di terra ${ }^{28} »$. Durante una delle nostre conversazioni scambiate all'interno del suo bureau, Sidi mi rivelò che in realtà la sua attività è anche riconosciuta formalmente dallo stato marocchino sotto il nome di Home Plast S.A.R.L. Era in possesso dei documenti che attestavano la dichiarazione di esistenza (i documenti erano in duplice copia in lingua francese e arabo fușhah) della sua goulssa e della sua mini-azienda, a livello formale, riconosciuta come una S.A.R.L (Società a responsabilità limitata). Nel bureau c'erano anche un telefono fisso, un fax e una stampante, ancora ricoperti da cellofan. Chiesi a Sidi per quale motivo erano ancora in quello stato e perché non li utilizzasse. Lui mi rispose ridendo: «Non funziono neanche io, figlia mia. E poi per lavorare non $\mathrm{mi}$ servono questi oggetti ${ }^{29}{ }^{\prime}$. Mi spiegò anche che la sua azienda non emette alcun fatturato poiché: «Ho pensato di fatturare all'inizio...ma secondo te...non posso certo chiedere le fatture ai bouâara! Questo lavoro si svolge senza carte, solo con la parola 
data. [...] Voglio le cose semplici e non complicate, altrimenti tutto questo non funzionerebbe ${ }^{30} »$.

\section{Conclusione}

19 L'attività di recupero è stata analizzata nella sua connessione con il settore formale poiché quest'ultimo, nonostante la complessa rete di dispositivi istituzionali e legislativi (Legge 28.00, Legge 54-05, PNDM, Gestion Déléguée, forze di sicurezza e militari) di cui gode, non è in grado di garantire una efficiente gestione dell'intera filiera. Categorie imprenditoriali con status e capacità economiche differenti vengono così assorbite al suo interno, creando un'industria di rigenerazione delle risorse dinamica e integrata all'economia urbana (Castells, Portes 1989). Il bisogno di materiali recuperabili e riciclabili è inesauribile: in questa loro fase, i rifiuti contenuti nei cassonetti dell'immondizia costituiscono una possibilità di azione e di ricavo: "per me non è spazzatura, almeno finché c'è qualcuno disposto a comprarla ${ }^{31}$ ". Recuperare oggetti abbandonati rappresenta un atto in grado di generare un'ecologia alternativa che attribuisce valore agli oggetti scartati e illustra come la condizione del rifiuto sia tutt'altro che una 'condizione finale' (Martínez 2017:349). È un atto che, se interpretato secondo il paradigma della 'giustizia spaziale' di Soja (2010), permette di rielaborare le condizioni sociali di appropriazione delle risorse localizzate, le molteplici relazioni di potere e l'articolazione delle identità e delle gerarchie territoriali. In questo senso il rifiuto viene quindi interpretato simultaneamente come una realtà materiale capace di influire su una determinata realtà sociale e come un elemento indispensabile di decifrazione di una grammatica simbolica di economia (in)formale. Integrare lo spazio come strumento di analisi e come prodotto sociale informa sul grado di visibilità dei processi presi in considerazione. La visibilità ha a che vedere pertanto con le politiche che caratterizzano la città di Casablanca e che determinano cosa è in o out of place (Cresswell 1996). Le forme di controllo sulle attività informali producono una asimmetria nella visibilità delle stesse, portando a una gerarchizzazione di sguardi diversi. La più visibile delle pratiche realizzate dagli chiffonniers si traduce in una dimensione temporale e spaziale ben precisa. L'azione di andare e percorrere la città viene quindi interpretata nel presente articolo come forma di intervento umano, fisico e simbolico sul paesaggio urbano. Una prassi che rafforza il loro legame con l'ambiente circostante. Riconoscendo gli interstizi di azione dove inserirsi, arrivando a compromessi e stringendo accordi interpersonali tra le amministrazioni, i commercianti e le grandi catene (Carrefour, Acima, La belle vie) i recuperatori consolidano il loro posizionamento nell'economia dominante.

La goulssa di Sidi è la prova di una relazione asimmetrica esistente tra gli attori sociali operanti nella filiera di gestione dei rifiuti in Marocco. Allo stesso tempo testimonia come questo microcosmo informale e locale sia direttamente o indirettamente ben inserito nelle filiere economiche istituzionalizzate, su scala locale, regionale, nazionale ed internazionale. Sidi, affermando che Lahraouine è un territorio abbandonato a sé stesso, implicitamente conferma questa tesi: le goulssa sono sottoposte così a un 'atto di riconoscenza' (Vallat 2001) e di tolleranza da parte dei poteri pubblici. Il muoversi in modo discreto e rapido degli chiffonniers lungo le strade trafficate di Casablanca, la recinzione delle goulssa costruita con materiali di fortuna che allontana la curiosità di sguardi indiscreti, la decisione di sidi di non richiedere fatture ai recuperatori 
marocchini si configurano quindi come pratiche locali, sociali e culturali, inscritte in un paradigma economico dominante internazionale (si pensi ai collegamenti con la Banca Mondiale). Rappresentano quindi esplicite intenzioni di appropriazione dello spazio urbano e indicano al tempo stesso l'intima conoscenza del luogo nel quale gli attori sociali presi in esame agiscono.

21 Metodologicamente, l'indagine etnografica sulla circolazione e sulla gestione dei rifiuti ha il potenziale di mettere in luce l'intreccio e l'interdipendenza tra pratiche ufficiali e ufficiose, spesso descritte come opposte le une alle altre. Casablanca ci dimostra che una città moderna rende visibile l'informalità al punto tale che separarla dal formale risulta un'operazione complicata poiché le due sfere si alimentano a vicenda. Grazie alla ricerca etnografica è emersa una vera e propria infrastruttura materiale e sociale, dove chiffonniers, mediatori, grossisti, istituzioni e imprese appartenenti al settore privato operano parallelamente, talvolta cooperando, dando vita a forme collettive di agentività politica entro le quali ogni attore è ben consapevole del proprio margine di azione.

In conclusione l'informalità alimenta l'economia formale (e viceversa) creando molteplici collegamenti e reti nello spazio urbano: i recuperatori di rifiuti sanno di potersi muovere per la città perché sono socialmente accettati, conoscono bene il loro obiettivo e le modalità con cui raggiungerlo. Rispetto alla 'logica dell'abbandono' che generalmente colpisce chi manifesta una vicinanza fisica rispetto al rifiuto, lo chiffonnier marocchino supera questo tipo di rappresentazione, trovando un posto per sé e per i rifiuti.

\section{BIBLIOGRAFIA}

Appadurai A., 1986 The Social Life of Things: Commodities in Cultural Perspective, University Press, Cambridge.

Baud I., Schenk H., 1994 Solid waste management: modes, assessment, appraisals and linkages in Bangalore, Manohar, New Delhi.

Ben Jelloun T., 2011 La Rivoluzione dei Gelsomini. Il risveglio della dignità araba, Bompiani, Milano.

Calafate-Faria F., 2016 «Marginal Attachment and Countercycling in the Age of Recycling», in M. Lancione (a cura di), Rethinking Life at the Margins: The Assemblage of Contexts, Subjects, and Politics, Routledge, London.

Cattedra R., 1990 «Nascita e primi sviluppi di una città coloniale: Casablanca 1907-1930», in Storia Urbana, LIII: 127-180.

Cavé J., 2015 La ruée vers l'ordure. Conflits dans les mines urbaines de déchets, Presses universitaires de Rennes, Rennes.

Charton A., 1924 « Casablanca », in Annales de Géographie, XXXIII, (183): 303-307. 
Chen M., 2007 «Rethinking the Informal Economy: Linkages with the Formal Economy and the Formal Regulatory Environment», in DESA Working Paper (46), United Nations Department of Economic and Social Affairs, New York.

Compagnon A., 2017 « Les Chiffonniers de Paris», in Série Bibliothèque illustrée des histoires, Gallimard, Paris.

Cresswell T., 1996 In Place/Out of Place, University of Minnesota Press, Minneapolis, London.

Debout L., Florin B., 2011 « Les contradictions du nouveau système de déchets au Caire. Conflits, négociations et stratégies d'acteurs », in Égypte-Monde arabe, VIII : 31-57.

Douglas M., 1966 Purity and Danger: An Analysis of the Concepts of Pollution and Taboo, Routledge, London.

Douglas M., 1986 How institutions think, Syracuse University Press, Syracuse, New York.

Emiliani M., 2012 Medio Oriente. Una storia dal 1991 a oggi, Laterza, Roma-Bari.

Ghalloudi J., Zahour G., Talbi M., 2015 « Evaluation de la première expérience de gestion déléguée des déchets ménagers à Casablanca », in European Scientific Journal, XI, (2) : 237-263.

Gilbert C., Henry E., 2012 «La définition des problèmes publics : entre publicité et discrétion », in Revue française de sociologie, LIII, (1) : 35-59.

Godard X., Fatanzoun I., 2002 Urban Mobility for All, Swets \& Zeitlinger, Lisse.

Guha-Khasnobis B., Kanbur, R., Ostrom E. (eds.), 2006 Linking the Formal and Informal Economy: Concepts and Policies, Oxford University Press.

Hart K., 1973 «Informal Income Opportunities and Urban Employment in Ghana», in The Journal of Modern African Studies, XI (1): 61-89.

Herzfeld M., 2006 Antropologia. Pratica della teoria nella cultura e nella società, Seid, Firenze.

Lautier B., 1991 « Les travailleurs n'ont pas la forme. Informalité des relations de travail et citoyenneté en Amérique latine », in B. Lautier, C. Miras, A. de Morice (éd. par), L'État et l'informel, L'Harmattan, Paris: 11-76.

Lefebvre H., 1974 La Production de l'espace, Anthropos, Paris.

Martínez F., 2017 «Waste is not the end. For an anthropology of care, maintenance and repair» in Social Anthropology, XXV: 346-350.

Marx K., 1867 Il Capitale. Critica dell'economia politica, Unione Tipografico-Editrice, Torino.

Medina M., 2007 The world's scavengers: salvaging for sustainable consumption and production, Altamira Press, Plymouth.

Mubi Brighenti A., 2010 Visibility in Social Theory and Social Research, Palgrave Macmillan, United Kingdom.

Piasere L., 1991 I popoli delle discariche, CISU, Roma.

Pinna L., 2011 Autoritratto dell'immondizia. Come la civiltà è stata condizionata dai rifiuti, Bollati Boringhieri, Torino.

Rachik A., 2002 «Casablanca : politiques urbaines et pressions sociales », in NAQD, I, (16): 55-65.

Raoulx B., Gutberlet J., Tremblay C., 2009 « Dans les poubelles de Vancouver. Le recyclage comme support de socialisation », in Le Sociographe, XXIX: 69-81.

Reno J., 2015 «Waste and Waste Management», in Annual Review of Anthropology, (44): 557-72. 
Saitta P., Shapland J., Verhage A. (eds.), 2013 Getting By or Getting Rich? The Formal, Informal and Criminal Economy in a Globalized World, Eleven International Publishing, The Green Grass / Het groene gras Series, The Hague.

Schamber P. J., Suarez F., 2007 Recicloscopio, Miradas sobre recuperadores urbanos de residuos en América Latina, UNLA, Universidad Nacional de General Sarmiento.

Simmel G., 1903 «Die Grosstadt aund das Geistesleben», in Jahrbücher der Gehestiftung, (trad. it. in G. Martinotti, 1968 Città e analisi sociologica, Marsilio, Padova).

Soja E., 2010 Seeking spatial justice, University of Minnesota press, Minneapolis.

Tuan Y., 1979 «Space and place. Humanistic Perspective», in S. Gale, G. Olson (eds.) Philosophy in geography, D. Reidel Publishing Company, (20): 387-427.

Vallat C., 2001 « L'urbanisation illégale dans les grandes villes méditerranéennes: consolidation d'une pratique informelle», in Les très grandes villes dans le monde, Éditions du Temps, Paris: 105-132.

Vergara G., 2008 Género y pobreza: una aproximaciòn a las recuperadoras de San Franciso, CEA-UNC, Conicet, Universidad nacional de Cordoba.

Viale G., 1994 Un mondo usa e getta. La civiltà dei rifiuti e i rifiuti della civiltà, Feltrinelli, Milano.

Zaouaq M., Zaouaq K., 2019 «Le droit et les politiques applicables à la gestion des déchets plastiques au Maroc», in Journal du droit de l'environnement et du développement (LEAD Journal), XV, (2): 141-153.

\section{Sitografia}

Averda pagina web, averda.com. Consultato il 24/10/2020

www.averda.com.

Banca Mondiale, 2010 « La Banque Mondiale soutient la réforme du secteur de la gestion des déchets solides municipaux au Maroc», in Banque Mondiale.org. 20 Dicembre 2010. Consultato il 13/09/2017 www.banquemondiale.org/fr/news/press-release/2010/12/20/the-world-bank-supports-thereform-of-the-municipal-solid-waste-sector-in-morocco.

Bulletin Official n. 5480, «Legge 28.00 sulla gestione dei rifiuti e sulla loro eliminazione», in Aut.gov.ma. Consultato il 22/09/2018

http://aut.gov.ma/pdf/Loi_n28-00_relative_a_la_gestion.pdf.

Chankou A., 2014 «Charte communale 1976. Privatiser la commune», in Maroc Hebdo, 29 maggio 2014. Consultato il 13/09/2017 www.maghress.com/fr/marochebdo/125503.

Charte Communale, 1976, «Bulletin officiel nº 3335», in Adala.justice.gov. Consultato il 15/01/2017

http://adala.justice.gov.ma/production/html/Fr/78870.htm.

Derichebourg pagina web, derichebourg-maroc.com. Consultato il 24/10/2020 www.derichebourg-maroc.com/fr/accueil.

Haut-Commissariat au Plan, 2014 « Recensement général de la population et de l'habitat 2014 », in Hcp.ma. Consultato il 23/04/2019 www.hcp.ma/regcasablanca/attachment/673830. 
Ilo, International Labour Organization, ilo.org. Consultato il 26/09/2019

www.ilo.org/global/lang--en/index.htm.

La tribune, 2017, «Collecte des déchets : Suez perd son contrat avec Casablanca », in La Tribune.fr. 19 Settembre 2017. Consultato il 02/01/2018

www.latribune.fr/entreprises-finance/industrie/energie-environnement/collecte-des-dechetssuez-perd-son-contrat-avec-casablanca-750584.html.

Map, Agence marocaine de Presse, mapnews.ma. Consultato il 07/10/2019

www.mapnews.ma/fr.

Paillards S., « Les 10 plaies de Casablanca \#5 : "Poubelle la vie, un feuilleton à la Casanegra" », in Yabiladi.com. 6 Settembre 2019. Consultato il 18/08/2020

www.yabiladi.com/articles/details/82779/plaies-casablanca-poubelle-feuilleton-casanegra.html.

Programme Nationale des déchets ménagers, pncl.gov.ma. Consultato il 4/02/2019

www.pncl.gov.ma/fr/grandchantiers/Pages/PNDM.aspx.

Suez Environnement, suez-africa.com. Consultato il 13/09/2020

www.suez-africa.com/fr.

Wiego, Women in Informal Employment: Globalizing and Organizing, wiego.org. Consultato il

$16 / 09 / 2020$

www.wiego.org/.

\section{NOTE}

1. Il termine, letteralmente 'straccivendolo', indicava una figura dedita al recupero, alla selezione e alla pulizia di materie prime quali vecchi tessuti, ossa, cuoio, vetro e metalli (Pyat 1847). Attualmente in Marocco l'espressione viene utilizzata per fare riferimento a coloro che si dedicano alla raccolta, selezione e vendita di rifiuti (principalmente carta, cartone, plastica, metallo e rame).

2. Riassumendo, le analisi sulle economie informali sono accomunate dai seguenti elementi: - la sfera istituzionale dominante può ignorare l'esistenza di pratiche informali essendo incapace di integrarle nel suo tessuto, oppure tollerarle tramite meccanismi di co-operazione nei quali i burocrati risultano coinvolti in traffici illegali (si tratta in questo caso di un'evidente scelta politica che mira a limitare i danni derivanti da una cattiva allocazione delle risorse); - la burocrazia non riesce a integrare la sfera informale poiché tale manovra comporterebbe costi insostenibili e risulterebbe complicato agire su categorie imprenditoriali che hanno status e capacità economiche differenti (Saitta et al. 2013; Guha-Khasnobis et al. 2006).

3. Ispirandomi al pensiero di Mubi Brighenti (2007) e Cresswell (1996) la nozione di (in)visibilità non è pensata come un fenomeno monodimensionale o dicotomico. Al fine di evitare il rischio di un determinismo teorico, la interpreterò come un processo intrinsecamente ambiguo, legato a specifici contesti e reti sociali, tecniche e politiche che potrebbero essere definite 'regimi di visibilità' (Brighenti 2007: 3).

4. La nozione di giustizia spaziale emerge prevalentemente nel dibattito contemporaneo grazie alle prospettive di studiosi come Lefebvre (1974) e Soja (2010) i quali affermano che tale idea può essere realizzata visibilmente nello spazio. L'elemento spaziale nella nozione di giustizia è stato concettualizzato principalmente da Henri Lefebvre attraverso la triade concettuale di spazio 'concepito' (come costruzione mentale dello spazio), spazio 'percepito' (oggettivo e concreto) e spazio 'vissuto' (una combinazione di spazio percepito e concepito). Il divario tra lo spazio vissuto delle pratiche quotidiane, lo spazio concepito di programmatori e progettisti e lo spazio 
percepito da chi lo abita, fornisce importanti indicazioni sul grado di ingiustizia che lo caratterizza. In altre parole, la giustizia spaziale non può essere raggiunta in modo astratto, poiché le stesse relazioni sociali si svolgono in determinati luoghi e temporalità.

5. Sin dalla sua teorizzazione nel dibattito antropologico grazie allo studioso Keith Hart nel 1973, l'economia informale è stata oggetto di molteplici prospettive di analisi. Grazie a un'indagine etnografica condotta nella periferia di Accra in Ghana, l'antropologo britannico ha coniato l'espressione informal income opportunities per descrivere tutte quelle attività radicate nel tessuto economico e sociale della città, ma che sfuggono a un controllo amministrativo. Mestieri dunque capaci di generare reddito per determinati attori sociali attraverso pratiche che oltrepassano e si muovono in modo parallelo ai binari dell'economia ufficiale. Il recente interesse verso l'economia informale è stato accompagnato da una significativa ri-concettualizzazione. A partire dagli anni '90, la Women in Informal Employment: Globalizing and Organizing (WIEGO), una rete composta da ricercatrici e ricercatori attivisti, ha lavorato congiuntamente con l'Organizzazione internazionale del lavoro (ILO) per ampliare la definizione di settore informale, includendo non solo quelle attività carenti di una regolamentazione legale, ma anche tutti quei rapporti di lavoro che sono insufficientemente regolamentati o protetti. In linea con questa corrente di pensiero, una recente pubblicazione accademica, Rethinking the Informal Economy: Linkages with the Formal Economy and the Formal Regulatory Environment (Chen 2012), suggerisce di intendere il fenomeno dell'informalità come comprensivo di un'eterogeneità di processi quali transazioni economiche, scambi sociali e commerciali che avvengono al di fuori (e aggiungo talvolta in modo parallelo o congiunto) rispetto alla regolamentazione e al controllo statale.

6. Per un quadro riassuntivo e dettagliato consiglio la lettura di Le droit et les politiques applicables à la gestion des déchets plastiques au Maroc (Zaouaq, Zaouaq 2019).

7. L'analisi dello statuto del PNDN mette in luce il carattere ambizioso del programma: tasso di raccolta pari al $90 \%$ da raggiungere entro il 2020 , riqualificazione e chiusura di tutte le discariche abusive esistenti sul territorio marocchino, sviluppo di una filiera selezione-riciclovalorizzazione dei rifiuti. Per maggiori dettagli si rimanda alla pagina web: www.pncl.gov.ma/fr/ grandchantiers/Pages/PNDM.aspx.

8. I nomi degli interlocutori e delle interlocutrici riportati nel saggio figurano come pseudonimi per motivi di privacy.

9. Note di campo del 26/11/2016. Hamid, Salè.

10. Il dominio coloniale francese in Marocco (1919-1956) ha rappresentato una tappa generatrice di molteplici ripercussioni a livello sociale, linguistico, economico e politico ed urbanistico, con effetti visibili tuttora nello spazio urbano di Casablanca. Due processi in particolare hanno cambiato radicalmente il volto della città: il potenziamento delle linee ferroviarie e stradali che ha prodotto una considerevole migrazione interna di abitanti dalle zone rurali verso la città (Charton 1924) e un processo cumulativo di iper-urbanizzazione (Cattedra 1990; Rachik 2002) con una conseguente e incontrollata estensione della periferia a sud e sud-est della di Casablanca e diffusione di insediamenti informali definiti bidonville.

11. Prima di questa decisione, la gestione pubblica della nettezza urbana era una responsabilità che gravava esclusivamente sui comuni: la Charte Communale del 30 settembre 1976, tramite un processo di decentralizzazione dei poteri, affidava la gestione del piano di risanamento di ciascun comune del paese alle collettività locali (Bulletin Officiel 1976). Il consiglio comunale era quindi a capo della creazione e dell'organizzazione dei servizi pubblici comunali e della loro gestione. Tuttavia emersero ben presto le falle di questo sistema che «rispondeva sempre meno alle esigenze di una democrazia locale efficace e moderna» (Chankou 2014). La carta comunale del '76 presentava importanti limiti nella realizzazione dei piani comunali a causa della supervisione del Ministero dell'Interno sui consigli comunali, esercitata in modo particolare sulle questioni finanziarie, con notevoli ritardi nell'espletamento delle procedure amministrative comunali.

12. Corrispondenti a circa $€ 920$ e $€ 184.000$. Il testo è tradotto dal francese da chi scrive. 
13. La gestione delegata è un contratto in base al quale una persona giuridica di diritto pubblico, nota come 'delegante', delega per un periodo di tempo limitato la gestione di un servizio pubblico di sua competenza a una persona giuridica di diritto pubblico o privato, denominata 'delegato' riconoscendole il diritto a ricevere la remunerazione da parte degli utenti e /o a trarre profitto da detta gestione (Ghalloudi et al 2015).

14. Queste appellativi sono emersi durante il fieldwork, nelle conversazioni informali con i miei interlocutori e da un'analisi della stampa digitale (Paillard 2019), consultata sul tema.

15. Attualmente le due società che si sono aggiudicate gli appalti per la nettezza urbana di Casablanca sono la multinazionale libanese Averda e la francese Derichebourg.

16. Attualmente il reclutamento del moqaddem avviene in assenza di norme giuridiche, sulla base di un processo di cooptazione e di garanzie che questi agenti assicurano per assumere i compiti che verranno loro dati in qualità di 'responsabile di quartiere'.

17. Il termine francese, che deriva da chiffon, ovvero stracci, cenci. In dārija l'origine del termine bouaâra (bouâar al singolare) è incerta e sensibile di possibili interpretazioni. Avanzo l'ipotesi

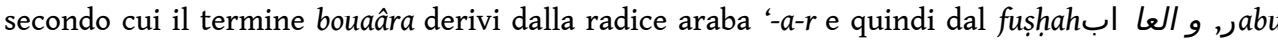
al-'ar, letteralmente 'il padre della vergogna', inteso quindi come colui che compie atti senza un minimo di pudore. In seguito l'espressione passando alla pronuncia dialettale si è contratta, diventando così l-bouara (i bouâara). Per evitare espressioni dispregiative, utilizzerò il termine chiffonnier.

18. I grossisti proprietari delle goulssa vengono chiamati con il termine francese grossistes oppure nel dialetto marocchino sono definiti moul al-goulssa letteralmente il proprietario della goulssa.

19. Il termine goulssa presenta dei connotati culturalmente densi in quanto deriva dalla radice araba 'g-l-s' che designa l'atto di sedersi. Goulssa designa pertanto una 'seduta' o anche una 'sessione'. Emerge in tal senso che il nome dato a questo spazio rispecchia il tipo di attività che viene svolto al suo interno, in contrasto soprattutto con l'attività dell'andare e del camminare, stando in piedi, dei recuperatori informali lungo le principali arterie di Casablanca.

20. Note di campo del 24/11/2016. Zakarya, pittore, Casablanca.

21. L'analisi del contesto istituzionale e politico ci porta ad avanzare l'ipotesi che l'atteggiamento di tolleranza, di laisser faire da parte delle istituzioni e delle forze dell'ordine, siano il sintomo di una forte tensione tra una popolazione in continuo fermento e la necessità da parte delle istituzioni, e della monarchia in primis, di tenerla a bada per timore di possibili ondate rivoluzionarie (come è già avvenuto in altri paesi del Maghreb come Tunisia ed Egitto). Si ricordi che tuttora in Marocco aleggia lo spettro di eventi drammatici che hanno destabilizzato gli scenari politico-sociali non solo in Marocco, ma in tutto il nord Africa. Per un approfondimento, si consiglia la lettura di "La Rivoluzione dei Gelsomini. Il risveglio della dignità araba" di Tahar Ben Jelloun (2011).

22. Note di campo del 07/12/2016. Khalid, recuperatore informale, Casablanca.

23. Note di campo del 05/05/2017. Simo, recuperatore informale, Salé.

24. Note di campo del 28/04/2017. Sidi, Casablanca.

25. Note di campo del 28/04/2017. Sidi, Casablanca.

26. È necessario fare un accenno al ruolo che la circolazione dei rifiuti ricopre nella definizione di uno spazio come centro e/o periferia. I luoghi che accolgono gli scarti sono in genere «distanti da quelli in cui si svolgono le principali attività economiche e culturali» (Calafate-Faria 2016). A questo punto però, sorge una domanda. Su quali basi e a quali condizioni uno spazio viene utilizzato per ospitare i materiali di scarto? Cosa lo caratterizza rispetto ad altri spazi? E qual è la connessione tra la circolazione dei rifiuti e la nozione di giustizia spaziale. L'antropologo Reno (2015) ci aiuta nella risposta, parlando di political removal and emptying spaces: luoghi in cui arrivano i rifiuti perché hanno spazio sufficiente per essere riempiti con materiali di scarto e perché mancano dei mezzi necessari per resistere e far fronte all'arrivo di materiale impuro. Le 
industrie inquinanti, ad esempio, sono spesso posizionate in aree che forniscono per una serie di ragioni minore resistenza politica (Ibidem).

27. A seguito dell'improvvisa crescita demografica di Casablanca, sono presenti nella città numerosi quartieri nati e sviluppatisi senza un vero e proprio piano di urbanizzazione. Di conseguenza gli insediamenti non dispongono dei principali servizi pubblici.

28. Note di campo del 28/04/2017. Sidi, Casablanca.

29. Note di campo del 28/04/2017. Sidi, Casablanca.

30. Note di campo del 26/04/2017. Sidi, Casablanca.

31. Note di campo del 18/01/2017. Naoufeq, recuperatore informale, Salé.

\section{RIASSUNTI}

Questo articolo presenta i risultati di una ricerca etnografica condotta a Casablanca (Marocco) tra il 2016 e il 2017. L'obiettivo è illustrare le modalità di circolazione dei rifiuti tra i differenti attori sociali protagonisti della scena culturale analizzata: recuperatori informali, grossisti e istituzioni. Decostruendo una visione univoca del mestiere del recuperatore, proporrò una descrizione fluida della sua agency, in costante tensione con le istituzioni che dominano la governance dei rifiuti. Il focus sulle interazioni socio-materiali mi condurrà verso un riposizionamento della nozione di informalità e di rifiuto, interpretati entrambi come risorse motrici di forme di condivisione tra persone, luoghi e oggetti, in uno scenario locale in cui emergerà la complementarietà tra pratiche ufficiali e ufficiose.

This paper illustrates the findings of an ethnographic research conducted in Casablanca (Morocco) between 2016 and 2017. The aim is to shed light on waste circulation between the main social actors of the analysed cultural scenario: informal waste-pickers, wholesalers and institutions. By de-constructing the garbage collectors' single-narration, I will propose a fluid description of their agency, in a constant tension with the institutions that dominate wastegovernance processes. The focus on the socio-material interactions will lead me towards a repositioning on the very notions of informality and waste, both interpreted as driving resources of sharing processes between people, places and objects, in a local scenario where the complementarity between official and unofficial practices will stand out.

\section{INDICE}

Parole chiave : giustizia spaziale, rifiuti, recuperatori urbani, informalità, economie informali Keywords : spatial justice, waste, urban waste-pickers, informality, informal economies

\section{AUTORE}

\section{ANNA KARIN GIANNOTTA}

Dipartimento di Scienze Storiche, Geografiche e dell'Antichità, Università degli Studi di Padova annakarin.giannotta@studenti.unipd.it 